\title{
Coronary Artery Disease Awareness: Levels of Socioeconomic Status and Dietary Restriction
}

Snehita Prasad, ${ }^{1}$ Mritunjay Kumar Mishra, ${ }^{2}$ Tapeshwar Yadav $^{3}$

${ }^{1}$ Department of Biochemistry, Narsinhbhai Patel Dental College and Hospital, Sankalchand Patel University, Visnagar, Gujarat, India; ${ }^{2}$ Department of Biochemistry, Smt. BK Shaha Medical Institute and Research center, Sumandeep Vidyapeeth University, Vadodara, Gujarat, India; ${ }^{3}$ Department of Laboratory Medicine, Madan Bhandari Academy of Health Sciences, Hetauda, Makwanpur, Nepal.

Correspondence:

Snehita Prasad. Email: snehitaprasad@gmail.com

Introduction: - India has undergone a remarkable demographic transition over the period time. Socioeconomic status indicators including education, income, and occupation are associated with coronary heart disease risk factors, morbidity, and mortality.

Objective: - To assess the socioeconomic status and diet restriction in patients with coronary artery diseases.

Methods: - The present study was a type of observational study conducted on total 200 subjects in Dhiraj Hospital, Sumandeep Vidyapeeth from August-2019 to July-2020 after ethical approval. Out of which 100 were coronary artery diseases and 100 were healthy controls. Patients with other than coronary artery diseases were excluded from the study, data were collected with the help of a pre-validated questionnaire and analyzed by SPSS-16 software.

Results: - Eighty-nine subjects with coronary artery diseases and seventy-five healthy controls were physically inactive. Eighty-five subjects with coronary artery diseases do not have any diet restriction, however, fifty-nine subjects had exposure to only primary education.

Conclusion: - Majority of the subjects were from a rural area, there were high illiteracy levels so there was low awareness. Patients could come to know the importance of physical activities and how to maintain a normal weight by following a dietary regimen to minimize the risks of disease.

Keywords: - Awareness; coronary artery disease; education; occupation; physical activity.

Submitted: May 22, 2021 Accepted: June 22, 2021 Published: June 28, 2021

\section{INTRODUCTION}

Coronary artery disease is one of the main worldwide health risks. This disease is one of the leading causes of mortality in the world. ${ }^{1}$ Regular physical activity and exercise are important in reducing the risk of death and cardiovascular events in primary as well as in secondary prevention. ${ }^{2}$ A sedentary lifestyle is one of the major risk factors for cardiovascular diseases (CVD). India has a huge burden of coronary artery diseases
(CAD) and efforts to promote physical activity and reduce sedentary lifestyle plays an important role in containing the problem. ${ }^{2}$ Socioeconomic inequalities in health can be defined as systematic differences in the prevalence or incidence of health problems between people of higher and lower socioeconomic Citation: Prasad S, Mishra MK, Yadav T. Coronary artery disease awareness: levels of socioeconomic status and dietary restriction. Nepal J Health Sci. 2021 Jan- Jun;1(1): 37-42 
distinguished by the level of education, occupational class, or income. ${ }^{3}$ Diet plays an important role both in physiology and pathology. It has an important role to maintain health, causation of disease, progression of the disease, and also recuperating from unhealthy status to healthy. It is considered to be another more efficient and effective way in the management of CVD. ${ }^{4}$

Looking into the above aspects, the study was planned to determine the socio-economic strata (occupation, education, physical activity) and diet restriction with a level of knowledge in case control with patients of coronary artery disease.

\section{METHODS}

The present study was conducted in the department of biochemistry, Smt. BK Shah Medical Institute and Research center (SBKSMI \& RC), Sumandeep Vidyapeeth from August 2019 to July-2020. This is a case-control and observation type of study. A total 200 subjects of either sex having an age group of 40-70 years were enrolled for this study. Out of which 100 patient's coronary artery diseases and 100 healthy controls without age sex matched were taken for study. The data for this study were collected from the department of cardiology, Dhiraj Hospital, SBKSMI \& RC, and Sumandeep Vidyapeeth. The study was approved by the institutional ethical committee (SVIEC/ON/Medi/BNPG19/D20066). After getting written consent from every individual, data were collected regarding socioeconomic status like occupation, education, and physical activity were recorded by pre-validated questionnaire through the sequence wise interview session. Each subject was interviewed for the consumption of all kinds of foods and beverages using dietary recall in the hospital and home. Dietary intake patterns and timing for food intake of individuals were also recorded. Subjects with proven coronary artery diseases were enrolled for this study. The enrolment of the patients was made after the critical review done by a cardiologist based on clinical assessment and evaluation of cardiac biomarkers. Subjects of the known case of diabetes mellitus, liver diseases, kidney diseases, any nutritional disorder, and other than CAD were excluded from the study. Recorded data were presented in percentages, pie charts, and graphs. For categorical data presentation, frequency tables were used.

\section{RESULTS}

A case-control study with 100 patients with coronary artery diseases and 100 healthy controls was planned to observe the socioeconomic status including physical activity occupation, education, dietary awareness among the rural area population. Observed physical activity was categorized into light, moderate, vigorous, and physically inactive and we found that there was a higher number of subjects were fall under physically inactive. In regards to occupation and education, we have noted the type of profession such as government, private or business, etc. whereas in education, we categorize a level of education into primary, secondary, higher secondary, and graduate, where we found a higher number of subjects having only primary education. However, in dietary patterns, we found that many people who were used to avoid junk food like pizza, burgers, cheese, ghee, etc.

Table 1: Physical activity-based distribution in case and control groups

\begin{tabular}{l|c|c|}
\multicolumn{1}{c|}{ Physical activity } & \multicolumn{2}{|c|}{ Frequency } \\
\cline { 2 - 3 } & Case (n=100) & Control (n=100) \\
\hline $\begin{array}{l}\text { Light physical activity (Yoga, Meditation \& } \\
\text { light physical workout) }\end{array}$ & $09(9 \%)$ & $25(25 \%)$ \\
\hline $\begin{array}{l}\text { Moderate physical activity (Fast walking, } \\
\text { Swimming \& Weight lifting) }\end{array}$ & $01(1 \%)$ & $0(0 \%)$ \\
\hline $\begin{array}{l}\text { Vigorous physical activity (Jogging, running, } \\
\text { sports activity) }\end{array}$ & $01(1 \%)$ & $0(0 \%)$ \\
\hline $\begin{array}{l}\text { Physical inactive } \\
\text { Phy }\end{array}$ & $89(89 \%)$ & $75(75 \%)$
\end{tabular}


The physical activity was categorized into light, moderate, vigorous and others groups and the Total number of physically inactive subjects are $89 \%$ in case group and $75 \%$ in control group. The subject who performs light physical activity every day is $9 \%$ and moderate is $1 \%$ and vigorous is $1 \%$ total $11 \%$ subject perform physical activity in case group and total $25 \%$ in control group. (Table 1 )

Table 2: Occupation based distribution in case and control groups

\begin{tabular}{|l|cc|}
\hline \multirow{2}{*}{\multicolumn{1}{c|}{ Occupation }} & \multicolumn{2}{c|}{ Frequency } \\
\hline Not employed & Case (n=100) & Control(n=100) \\
\hline Government employee & $(15 \%)$ & $34(34 \%)$ \\
\hline Private employee & $02(2 \%)$ & $01(1 \%)$ \\
\hline Business & $17(17 \%)$ & $05(5 \%)$ \\
\hline Agriculture & $04(4 \%)$ & $10(10 \%)$ \\
\hline Labors/daily wages & $56(56 \%)$ & $36(36 \%)$ \\
\hline
\end{tabular}

Occupation based distribution frequency for both study subjects and corresponding controls. There were $15 \%, 2 \%, 17 \%, 4 \%, 56 \%, 6 \%$ subjects belong to not employed, government employee, business class, agriculture, labors/ daily wages respectively. In the control group the corresponding number of different religion groups was $34 \%, 1 \%, 5 \%, 10 \%, 36 \%, 14 \%$ respectively. The distribution occupation in both the groups and corresponding number is also presented in the form chart. (Table 2)

Table 3: Education based distribution in case and control groups

\begin{tabular}{|l|cc|}
\hline \multirow{2}{*}{ Education } & \multicolumn{2}{c|}{ Frequency } \\
\cline { 2 - 3 } & Case (n=100) & Control (n=100) \\
\hline Illiterate & $36(36 \%)$ & $30(30 \%)$ \\
\hline Primary & $45(45 \%)$ & $51(51 \%)$ \\
\hline HSC & $06(6 \%)$ & $11(11 \%)$ \\
\hline SSC & $08(8 \%)$ & $04(4 \%)$ \\
\hline Graduate & $05(5 \%)$ & $04(4 \%)$ \\
\hline
\end{tabular}

Education based distribution frequency for both the study subjects and corresponding controls. There were $36 \%, 45 \%, 6 \%, 8 \%$ and $5 \%$ subjects belong to illiterate, primary, HSC, SSC, graduate respectively. In the control group the corresponding number of different religion groups was $30 \%, 51 \%, 11 \%, 4 \%$, and $4 \%$ respectively. The distribution education groups and corresponding number is also presented in the form chart.(Table 3)

\section{Diet Restriction}

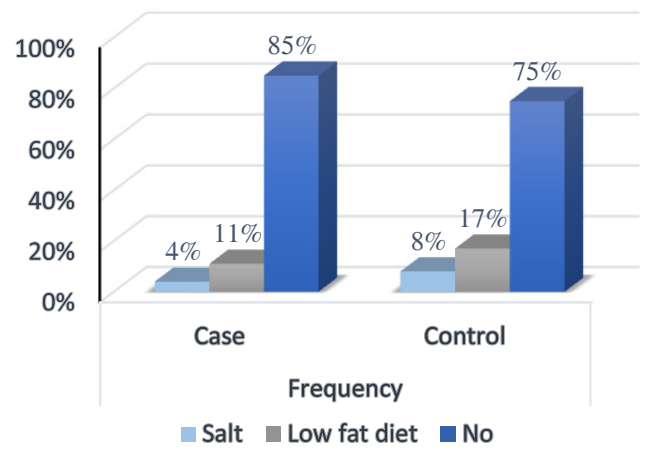

Avoiding diet during regularr outine

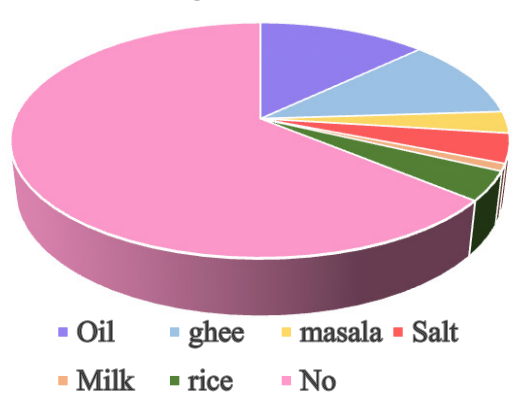

Figure 1: Awareness of diet restriction for study subjects and corresponding controls 
Figure 1 shows awareness about follow up their diet restriction for both study subjects and corresponding controls. The subject who was aware with diet restriction of case group include salt restriction is $4 \%$, low fat diet is $11 \%$ and $85 \%$ individuals were not follow their diet restriction respectively. Similarly in control group percentage of salt restriction is $8 \%$, low fat diet is $17 \%$ and $75 \%$. Subjects who were avoided their regular dietary pattern on routinely basis are: oil (13\%), ghee (11\%), masala (3\%), salt (4\%), milk (1\%), rice (4\%) and $64 \%$ individuals were not avoided these food items in their daily regular pattern. In the corresponding controls.

\section{DISCUSSION}

Cardiovascular diseases especially coronary artery diseases are the major contributing factors that are responsible for the deaths that occur in the global population among the non-communicable diseases..$^{5,6}$ coronary artery disease is caused by atherosclerosis of the coronary arteries and is the leading cause of mortality and morbidity among the various rural areas of developing countries. ${ }^{7}$ Lack of an active lifestyle is known to be one of the major risks of the development of CVD including CAD. Scientific evidence strongly proves that physical activity is associated with at least a two-fold increase in the risk for coronary events. ${ }^{2}$ The socioeconomic status of the individuals determines how a person adopts lifestyle and social status, which is directly determined by the economic status of individuals and family. Physical activity is a key determinant for energy balance and weight control. ${ }^{8}$ So, the present study was planned to assess socioeconomic characteristics and dietary awareness among 100 coronary artery disease patients and 100 healthy controls.

Physical activity (PA) is one of the key determinants to independently decreases the risk for coronary heart disease. Any aerobic exercise, such as walking, swimming, dancing, and lighter yoga can strengthen heart muscles and that makes the heart pump the required amount of blood to every cell and tissue. ${ }^{9}$

The present study observed the physical activity between cases and controls and it was observed a majority of individuals were physically inactive in cases as well as controls. In the subjects out of $11 \%$ were doing some physical activity $9 \%$ are the low was doing a light physical workout like yoga, meditation and $1 \%$ in each were involved in moderate or vigorous physical activity. All the control subjects who are involved in some form of physical activity that was light activity and were not involved in moderate and vigorous physical activity (Table-1). In the present study, it can be clearly said that the rural population who are visiting this hospital is found to be mostly following a sedentary lifestyle. More number of controls were involved in some sort of physical activity compared to CAD subjects.

Vanderploag. et al. and his colleague has conducted on Australian adults and reported that prolonged sitting or sedentary lifestyle was associated with increased risk for diabetes and $\mathrm{CAD}$. A benefit of regular exercise in preventive cardiovascular events was reported in a review. The study shows an inverse association between physical activity and all causes of mortality and active individuals reportedly have approximately a 30\% lower risk of dying during follow-up compared with active individuals. ${ }^{10}$ However, Zachariah et al. had observed a similar kind of pattern with low physical activity. ${ }^{2}$

Knowing about the disease and its implications improves with the increase with the levels of the education, people who are educated tend to have more awareness about the various aspects of the disease, nutritional regimens to be followed and importance of exercise, etc. Illiteracy plays a significant role in the contribution of high risk of cardiovascular diseases including coronary artery diseases and coronary heart diseases, especially in rural populations. The high levels of illiteracy and also low levels of education is the most contributing factor in the prevalence and progression of diseases.

Looking into the above fact, we have surveyed both the study subjects and controls in the aspects such as occupation and education. We observed that the majority of subjects were agriculture farmers both in cases and controls, whereas $34 \%$ were not employed in cases and $15 \%$ in controls. However, $10 \%$ cases and $4 \%$ in controls are doing some sort of business and $6 \%$ and $14 \%$ were the laborers or daily wages (Table-2). 
Around $59 \%$ of study subjects had some exposure to the education may be primary, SSC, HSC in the study subjects is in the corresponding control subjects $66 \%$. In the study subjects, $5 \%$ were graduates and in controls $4 \%$ were graduates and whereas remaining $36 \%$ and 30\% in both the study subjects and controls did not have any sort of education (Table-3).

Diet is an important risk factor for coronary heart disease. Food-related risk factors include obesity, high blood pressure, uncontrolled diabetes, and a diet high in saturated fats. A low-saturated fat, high-fiber, high plant food diet can substantially reduce the risk of developing heart disease.11

Due to lack of awareness about the benefits of restrictions of a salt, low-fat diet, we have noted that the majority of patients and controls did not have any awareness about diet restrictions such as salt and a low-fat diet. However, $64 \%$ of the subjects and 50\% of the control did not avoid diet with high, ghee, and masala contents (Figure 1). A link between dietary intake of fruit, vegetables, and whole grains and protection against coronary heart diseases has been reported. Dietary patterns have frequently been classified as two patterns: "healthy or prudent" and "Western." In general, the "healthy" pattern has been associated with more favorable biological profiles, slower progression of atherosclerosis, and a reduced incidence of coronary heart diseases compared with the "Western" pattern. ${ }^{12}$
The present study has limitations with a lower sample size and descriptive data. We have also not evaluated any biochemical markers in this study to see their relationship. However, the descriptive data needed a large sample size with biomarker evaluation.

\section{CONCLUSIONS}

The present study has highlighted the information regarding personal details such as social, educational status, dietary practices, lifestyles observed, awareness levels regarding the disease. From this it can be presented that the majority of the subjects did not know about the dietary regimen to be followed, physical activity to be observed and habits to be discontinued. The benefit of the present study is that patients could come to know the importance of physical activities and how to maintain a normal weight by following a dietary regimen to minimize the risks of disease.

\section{ACKNOWLEDGEMENT}

I would like to thank the research cell of Sumandeep Vidyapeeth for providing me the facility to conduct the research. I also want to thanks faculty members of Biochemistry, Sumandeep Vidyapeeth for their timeto-time cooperation and support.

\section{Conflict of interest: None}

\section{REFERENCES}

1. Attarchi, M., Mohammadi, S., Nojomi, M., \& Labbafinejad, Y. (2012). Knowledge and practice assessment of workers in a pharmaceutical company about prevention of coronary artery disease. Acta Medica Iranica, 50(10), 697-703. [ PubMed]

2. Danyang Tian, Jinqi Meng. Exercise for prevention of cardiovascular disease: Evidence-based recommendations. Oxid Med Cell Longev. 2019; 2019: 3756750. [PubMed | DOI ]

3. Mackenbach JP, Stirbu I, Roskam AJ, Schaap MM, Menvielle G, Leinsalu M, Kunst AE; European Union Working Group on Socioeconomic Inequalities in Health. Socioeconomic inequalities in health in 22 European countries. N Engl J Med. 2008;358(23):2468-81. [PubMed |DOI ]

4. Rambabu Kondredddy, Ali Chenak,Uma Shankar Akula, Addison garabet, Shakila Srikumar, Abdalla M Jarari, Jagannadha Rao Peela. (2012). Study of Lipid Profile in Coronary Heart Disease patients in Libya Journal of biomedical sciences, 2012; 1(4):3. [DOI]

5. O’Donnell MJ, Chin SL, Rangarajan S, Xavier D, Liu L, Zhang H, et al. Global and regional effects of 
1. potentially modifiable risk factors associated with acute stroke in 32 countries (INTERSTROKE): a case-control study. Lancet. 2016 Aug;388 (10046):761-75. [PubMed| DOI]

2. Dehghan M, Mente A, Teo KK, et. al. Relationship between healthy diet and risk of cardiovascular disease among patients on drug therapies for secondary prevention: a prospective cohort study. Circulation. 2012; 126:2705-2712.[ PubMed | DOI ]

3. Mritunjay Kumar Mishra, ZG Badade1, SK Kaul, Roshan Kumar Mahat, Vedika Rathore. Ischemia Modified Albumin (IMA) and lipid profile in coronary artery disease with and without type 2 Diabetes Mellitus. Sch. Acad. J. Biosci., 2016; 4(8):609-612. [DOI]

4. Mishra Mritunjay, DVSS Ramavataram. Predisposing Risk Factors Associated with Acute Myocardial Infarction (AMI): A Review. Indian Journal of Forensic Medicine \& Toxicology. 2021;15 (2):406-417. [Full Text | DOI]

5. Mercedes R. Carnethon. Physical Activity and Cardiovascular Disease: How Much is Enough? Am J Lifestyle Med. 2009 Jul; 3(1 Suppl): 44S-49S. [PubMed | DOI]

6. van der Ploeg HP, Chey T, Korda RJ, Banks E, Bauman A. Sitting time and all-cause mortality risk in 222497 Australian adults. Arch Intern Med 2012; 172:494-500. [PubMed | DOI]

7. Harpal S Buttar, Timao Li, Nivedita Ravi. Prevention of cardiovascular diseases: Role of exercise, dietary interventions, obesity and smoking cessation. Exp Clin Cardiol. 2005 Winter; 10(4): 229-249. [PubMed]

8. Bhupathiraju SN, Tucker KL. Coronary heart disease prevention: nutrients, foods, and dietary patterns. Clin Chim Acta. 2011; 412 (17-18):1493-514. [PubMed | DOI] 